\title{
REAMED LOCKED INTRAMEDULLARY NAILING FOR STUDYING FEMUR FRACTURE AND ITS COMPLICATIONS
}

O. Yoshino ${ }^{1}$, J. Brady ${ }^{1}$, K. Young ${ }^{2}$, B. Hardy ${ }^{1}$, R. Matthys ${ }^{3}$, T. Buxton ${ }^{4}$, R. Appleyard ${ }^{5}$, J. Tomka ${ }^{5}$, D. Dabirrahmani ${ }^{5}$, P. Woodford ${ }^{2}$, M. Fadia 6 , R. Steck ${ }^{7}$, A. Quail ${ }^{8}$, R.G. Richards ${ }^{9}$ and Z.J. Balogh ${ }^{1, *}$

${ }^{1}$ Department of Traumatology, University of Newcastle and John Hunter Hospital, 2310 Newcastle, NSW, Australia

${ }^{2}$ Hunter Pathology Service, Department of Pathology, University of Newcastle and John Hunter Hospital, 2310 Newcastle, NSW, Australia

${ }^{3}$ RISystem AG, 7270 Davos Platz, Switzerland

${ }^{4}$ School of Medicine, University of Newcastle, 2310 Newcastle, NSW, Australia

${ }^{5}$ Department of Biomedical Sciences, Faculty of Medicine and Health Sciences, Macquarie University, 2109 Sydney, NSW, Australia

${ }^{6}$ Anatomical Pathology, Canberra Hospital and Australian National University, 2617 Canberra, ACT, Australia

${ }^{7}$ Institute of Health and Biomedical Innovation, Queensland University of Technology, 4072 Brisbane, QLD, Australia

${ }^{8}$ Department of Anaesthesia, University of Newcastle and John Hunter Hospital, 2310 Newcastle, NSW, Australia

${ }^{9} \mathrm{AO}$ Research Institute Davos, 7270 Davos Platz, Switzerland

\begin{abstract}
Morbidity associated with femur fractures in polytrauma patients is known to be high. The many unsolved clinical questions include the immunological effect of the fracture and its fixation, timing of fracture fixation, management of fracture non-union, effect of infection and critical size of bone defects. The aim of this study was to establish a clinically-relevant and reproducible animal model with regards to histological, biomechanical and radiological changes during bone healing. A custom-designed intramedullary nail with interlocking system (RabbitNail, RISystem AG, Davos Platz, Switzerland) was used for fixation, following femur fracture. New Zealand White rabbits were assigned to two groups: 1. closed fracture model (CF; non-survival model: $n=6$, survival model: $n=3$ ) with unilateral mid-shaft femur fracture created by blunt force; 2 . osteotomy model (OT; survival model: $n=14$ ) with unilateral transverse osteotomy creating femur fracture. There were no intraoperative complications and full-weight bearing was achieved in all survival rabbits. Significant periosteal reaction and callus formation were confirmed from 2 weeks postoperatively, with a significant volume formation $\left(739.59 \pm 62.14 \mathrm{~mm}^{3}\right)$ at 8 weeks confirmed by micro-computed tomography $(\mu-\mathrm{CT}) .2$ months after fixation, there was no difference between the osteotomised and contralateral control femora in respect to the maximum torque $(3.47 \pm 0.35 \mathrm{~N} \mathrm{~m} v s .3 .26 \pm 0.37 \mathrm{~N} \mathrm{~m})$ and total energy $(21.11 \pm 3.09 \mathrm{~N} \mathrm{~m} \times$ degree vs. $20.89 \pm 2.63 \mathrm{~N} \mathrm{~m} \times$ degree) required to break the femur. The data confirmed that a standardised internal fixation technique with an intramedullary nail for closed fracture or osteotomy produced satisfactory bone healing. It was concluded that important clinically-relevant studies can be conducted using this rabbit model.
\end{abstract}

Keywords: Rabbit, animal model, intramedullary nailing, fracture healing, non-union.

*Address for correspondence: Zsolt J. Balogh, Department of Traumatology, John Hunter, Hospital and University of Newcastle, Locked Bag 1, Hunter Region Mail Centre, 2310 Newcastle, NSW, Australia. Telephone: +61 249214259 Fax: +61 $249214274 \quad$ Email: zsolt.balogh@hnehealth.nsw.gov.au

\section{Introduction}

In blunt trauma, skeletal injury is one of the leading causes of long-term disability in the young population (Balogh et al., 2012). Femur fracture is a serious injury, causing significant short-term morbidity with potential long-term local complications, including delayed union and non-union (Tay et al., 2014). The high-energy and open fractures are frequently associated with infections and segmental bony defects (Al-Arabi et al., 2007; Court-Brown et al., 1998; Ktistakis et al., 2014). In addition, they can be a major physiological insult to injured patients with polytrauma, causing haemodynamic, respiratory and 
immunological systemic complications (Lasanianos et al., 2011; White et al., 2006). The timing of fixation and the potential staged management of femur fracture in physiologically-compromised, multiple-injured patients remains controversial. For the investigation of these crucial questions on femur fracture management and its associated complications, a clinically relevant, reproducible animal model of femur fracture and operative stabilisation is essential. While the acute and chronic consequences of femur fracture and its fixation are different in nature, the recommended method of choice for mid-shaft femur fracture is a locked, reamed intra-medullary nail (Forster et al., 2005). The aim of this paper is to describe a new laboratory rabbit model with a standard implant and a clinicallyrelevant technique for femoral shaft fracture fixation.

\section{Materials and Methods}

After approval from the Animal Care and Ethics Committee of the University of Newcastle, Australia (Approval No. A-136), New Zealand White Rabbits ( $n=23$, weight $2.4 \pm 0.2 \mathrm{~kg}$ ) were assigned to two groups: 1 . closed fracture model (CF, $n=9$ ), with unilateral mid-shaft femur fracture and intramedullary fixation; 2. osteotomy model (OT, $n=14$ ), with transverse mid-shaft femur fracture by osteotomy and intramedullary fixation. In the CF group, 6 rabbits were assigned to a non-survival CF model and 3 to a survival CF model. All rabbits in OT were assigned to a survival model. Rabbits were anaesthetised with $3.0 \%$ isoflurane (Laser Animal Health, Eagle Farm, Queensland, Australia) and bolus of propofol, orally-intubated with a $4.0 \mathrm{~mm}$ endotracheal tube (Portex ${ }^{\circledR}$, Smiths, Dublin, OH, USA) and mechanically-ventilated with $100 \%$ oxygen with respiratory rate of 60 and tidal volume of $25 \mathrm{~mL}$. After induction, intravenous fentanyl, as analgesia, was initiated at $10 \mu \mathrm{g} / \mathrm{h}$, with the dose increased to $14 \mu \mathrm{g} / \mathrm{h}$ for the fracture procedure and internal fixation. The concentration of isoflurane was 2.0-3.5\% during preparation, fracture procedure and subsequent surgery. During the surgical preparation, all animals received $4 \mathrm{~mL} / \mathrm{kg} / \mathrm{h}$ of Ringer's lactated (RL) as intravenous fluid maintenance volume. Vital parameters were continuously monitored during the experiment, including mean arterial pressure (MAP), peripheral oxygen saturation $\left(\mathrm{SpO}_{2}\right)$ and temperature. Arterial blood gases (ABG) were collected for the measurement of $\mathrm{pH}, \mathrm{PaCO}_{2} \mathrm{PO}_{2}$, base excess (BE), bicarbonate $\left(\mathrm{HCO}_{3}\right)$, haemoglobin concentration $(\mathrm{Hb})$ and haematocrit $(\mathrm{Ht})$ and analysed by the i-STAT analyser (Heska, Waukesha, WI, USA).

\section{Fracture creation}

A standardised technique for producing a femur fracture in rabbits was developed based on the socalled Einhorn model, which was first published on a study in male Sprague-Dawley rats in 1984 (Bonnarens and Einhorn, 1984). The fracture apparatus producing a closed femur fracture was a three-point bending system. The femur was positioned in a standardised fashion on the support stage. A weight $(4 \mathrm{~kg})$ was dropped from the top of the rod $(40 \mathrm{~cm})$ and the blade
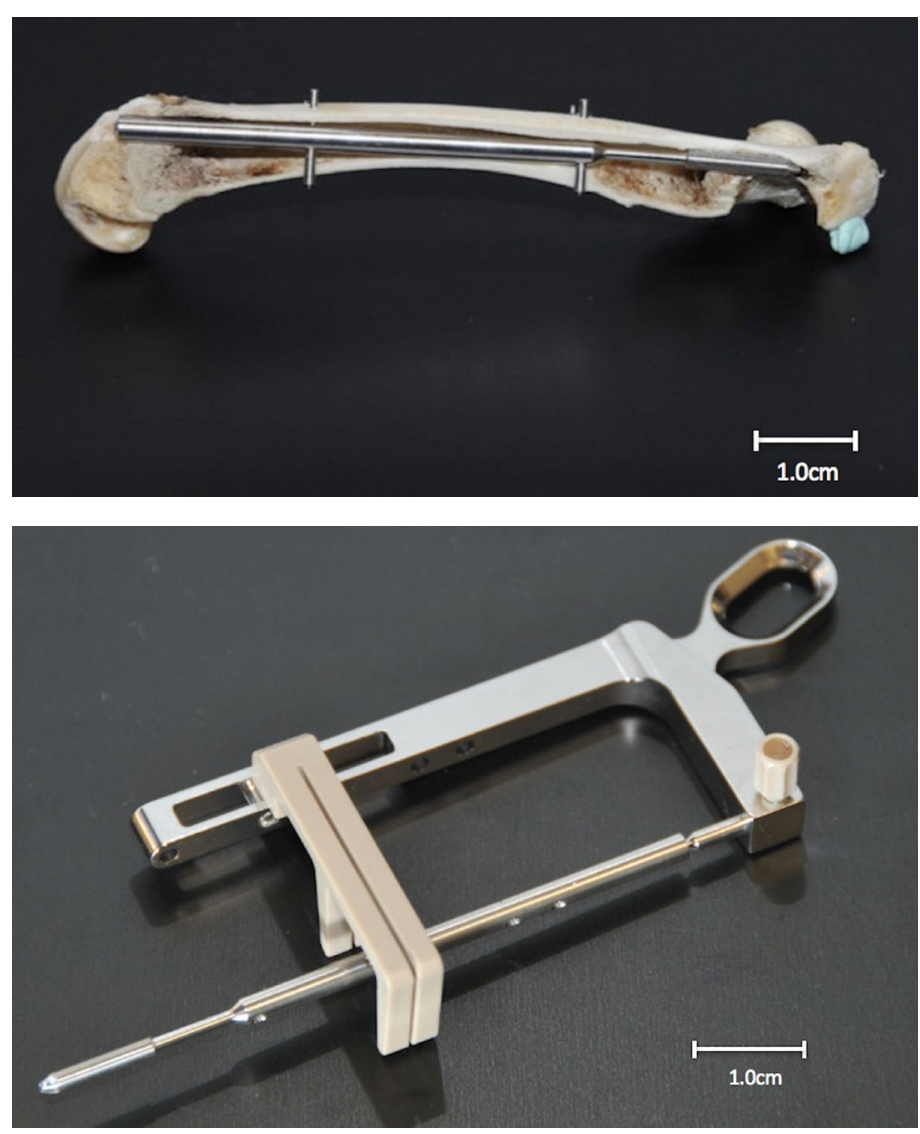

Fig. 1. RabbitNail in situ. The implant system contained a retrograde intramedullary nail that consisted of a distal shaft and a proximal threaded part with one proximal and two distal locking options $(\varnothing 1.00 \mathrm{~mm})$. The nail shaft was connected to the proximal threaded part by a flexible shaft to adapt to the curvature of the femur. The function of the proximal threaded part was to engage the nail into the cancellous bony area of the trochanteric region of the proximal femur.

Fig. 2. The locking screw insertion was facilitated by the specially-designed external aiming device and the device could be connected to the distal main shaft. A standard predefined osteotomy gap also may be created with a template for the guidance of a Gigli wire. 
descended midway between the two anvils, in order to produce a maximum bending moment precisely under the blade. As the magnitude of this moment can be calculated using mechanical principles, it is easily applied to the differing size of animals. This blunt mechanism for the production of femur fractures is similar to that of patients experiencing high-energy blunt force resulting in femur fractures, which is relevant to study polytrauma and its physiological and immunological consequences.

\section{Osteotomy creation}

The other method of fracture was by osteotomy using a $0.44 \mathrm{~mm}$ Gigli wire saw (RISystem AG, Davos Platz, Switzerland). A standardised sterile surgical technique was applied to all procedural steps. The rabbit was anaesthetised in the previously described manner and the lateral aspect of the thigh shaved, prior to the procedure. A $15 \mathrm{~mm}$ skin incision was made to expose the femur. Further incision was made through the underlying fascia with the scalpel and extended using a pair of scissors. Muscle was sharply separated along the later intermuscular septum to expose the femur. Once the femur was adequately exposed, the Gigli wire saw was applied and osteotomy, according to AO classification type A3 fracture (Muller, 1980), was completed. The value of this technique lies in its highly-reproducible fracture pattern, which is ideal for fracture healing, non-union and infection studies.

\section{Implants}

Based on an intact cadaveric rabbit femur, a new intramedullary nail and interlocking system with implant-specific instruments were designed (locking RabbitNail, provided by RISystem AG, Fig. 1 and Fig. 2) There were two versions for rabbits weighing around $2.5 \mathrm{~kg}$ and $3.0 \mathrm{~kg}$ (Fig. 3 and Fig. 4 ). The implant system contained a retrograde intramedullary nail [diameter $(\varnothing): 2.80 \mathrm{~mm}$ ] made of medical grade stainless steel 316L. The nail had one proximal and two distal locking options $(\varnothing 1.00 \mathrm{~mm}$ locking screws, made of medical grade stainless steel
316L). The locking screw insertion was facilitated by the specially-designed aiming device (Fig. 3). For the other option to create a standard predefined osteotomy gap, a template for the guidance of a Gigli wire saw was available (Fig. 2). The locking RabbitNail had a distal shaft $(\varnothing 2.80 \times 48.00 \mathrm{~mm})$ and a proximal threaded $(\varnothing 2.00 \times 12.00 \mathrm{~mm})$ part. In addition, a longitudinal borehole $(\varnothing 0.81 \times 3.00 \mathrm{~mm})$ was part of the proximal threaded end of the nail for enabling the engagement of a guide wire $(\varnothing 0.80 \mathrm{~mm})$, which facilitated the closed insertion technique of the retrograde RabbitNail into a broken femur. The distal shaft was connected to the proximal threaded part by a flexible shaft $(\varnothing 1.40 \times 8.00 \mathrm{~mm})$ to adapt it to the curvature of the femur. The function of the proximal threaded part was to engage the nail into the cancellous bony area of the trochanteric region of the proximal femur. The surgical fixation technique with the RabbitNail was extensively tested on plastic bone models and both intact and fractured cadaveric rabbit femora. Necessary adjustments were made on the implant and instruments until the above described final model was achieved. This was followed by the trial of the nail in non-surviving polytrauma models (Hardy et al., 2015) and finally on a surviving model of rabbit femur fracture and closed intramedullary nailing (described in the current paper).

\section{Internal fixation with intramedullary nailing}

After intravenous antibiotic prophylaxis (cephazolin, Sandoz, Macquarie Park, Australia: $20 \mathrm{mg} / \mathrm{kg}$ ), the rabbit was placed on the lateral position. The thigh was prepared by shaving the fur and chlorhexidine skin preparation. Standard sterile draping was performed and the principles of strict surgical asepsis were followed. Skin marking was performed over the patella, greater trochanter and the longitudinal line of the femur shaft, including the half-point between the patella and greater trochanter. A medial parapatellar arthrotomy was performed to expose the distal articular surface of the femur after the lateral dislocation of the patella. A $2.8 \mathrm{~mm}$ drill bit was used to open the medullary canal through the non-weight

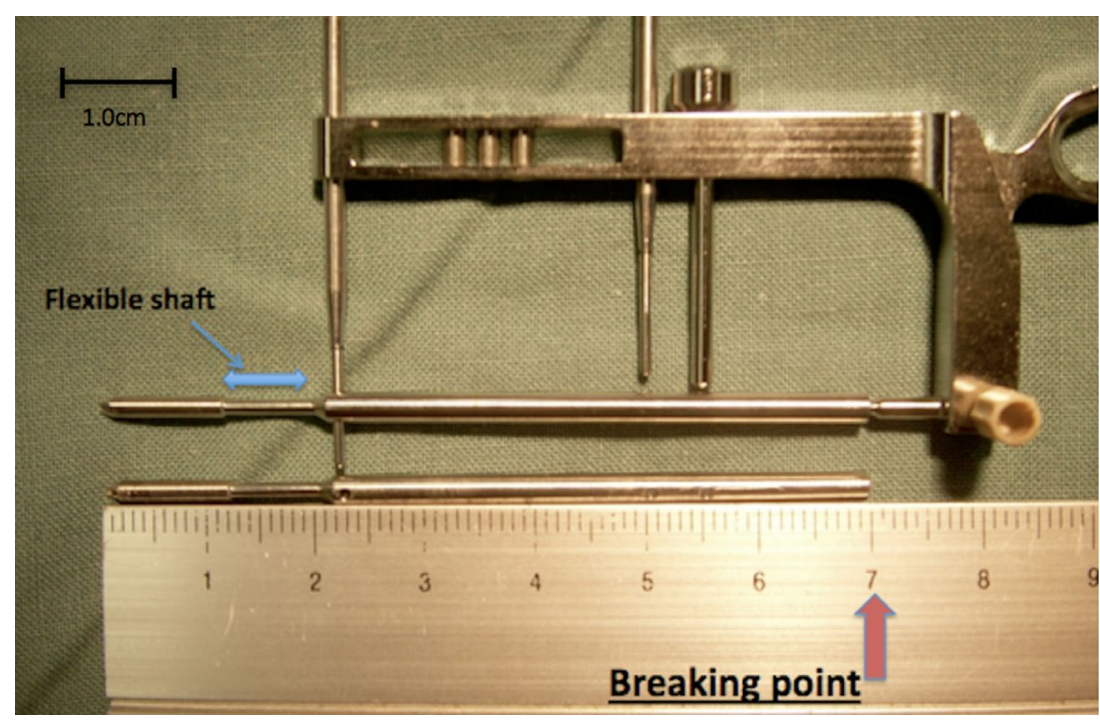

101
Fig. 3. RabbitNail with the external guide device. The RabbitNail consisted of a flexible threaded part ( $2 \mathrm{~mm}$ by $12 \mathrm{~mm}$ ) and a shaft ( $2.8 \mathrm{~mm}$ by $48 \mathrm{~mm}$ ). Up to three locking screws can be inserted through the guide device with small skin incisions. 
bearing surface of the distal femur articular cartilage. Reaming of the medullary canal was performed with a one-step $(2.8 \mathrm{~mm})$ reamer. A sharp $(\varnothing 2 \mathrm{~mm})$ stainless steel trocar was inserted into the medullary canal until its pointy tip penetrated the greater trochanter and its overlying soft tissues. A $300 \mathrm{~mm}$ flexible guide-wire was inserted through the trocar and the trocar removed. Fracture was created in the manner described above. After fractures were confirmed, the intramedullary nail (retrograde, locking RabbitNail) was introduced under the guidance of inserted wire that connected with the tip of the nail. Once the nail was in position, skin incisions were placed with an indication of the guiding device. A $1 \mathrm{~mm}$ drill bit, through the tissue-protecting sleeve (inserted into the aiming device), was used to make cortical holes for inserting locking screws. The locking pins were inserted by the single-use, pre-mounted screwdriver shafts with pre-defined breaking point. As soon as the screwdriver snapped off, the locking pin was fully interlocked and in position. The aiming device was removed, the wounds thoroughly irrigated with warm normal saline and capsular, subcutaneous and skin closure was performed with absorbable continuous sutures. Occlusive dressing and elastic bandage were applied.

\section{Perioperative care}

Perioperative care was conducted by the researchers and the animal care staff with the guidance of the institutional protocol. Subjects were transferred to a standardised animal care facility at the University of Newcastle at least $7 \mathrm{~d}$ before the experiments. Rabbits were isolated from each other by transparent walls and their weight, temperature, appearance, appetite, water and food consumption and wound condition monitored. If necessary, blood tests were conducted. Postoperatively, regular analgesia was provided with $4 \mathrm{mg} / \mathrm{kg}$ of carprofen for $2 \mathrm{~d}$ and $10 \mathrm{mg} / \mathrm{kg}$ of enrofloxacin for 1 week. After surgery and every week, radiographic evaluation of fracture healing was conducted. 8 weeks after surgery, rabbits were euthanised.

\section{Radiological imaging}

The experimental femora were radiographed weekly in anterior-posterior and lateral projections. Postoperative X-rays were taken to ensure fracture and correct osteotomy pattern and implant placement. AO fracture classification was applied (Muller, 1980). A Philips BuckyDiagnost $50 \mathrm{kV}$ generator with a Philips high-performance RO 1648 rotating anode tube was used (Philips, Eindhoven, The Netherlands). Images were taken with a $0.7 \mathrm{~mm}$ focal spot at a focal film distance of $110 \mathrm{~cm}$, an exposure of $50 \mathrm{kV}$ at $3.2 \mathrm{~mA}$ and an exposure time of $11.2 \mathrm{~ms}$. They were read using a Fuji Profect CS reader (Fujifilm, Tokyo, Japan) and an algorithm developed by Fuji for veterinary use and modified specifically for this research. Animals were sedated with propofol to facilitate X-rays.

\section{Radiographic scoring}

The score system adapted here was based on the previously published point system (An et al., 1999; Johnson et al., 1996; Yang et al., 1994). It consisted of the quantitative assessment of periosteal reaction, bone union and remodelling; the score could be between 0 and 10 (Table 1). Images were scored by an independent radiologist, who was blinded to the animal's treatment.

\section{$\mu-C T$ scan}

Animals were euthanised at 8 weeks with an intravenous injection of $300 \mathrm{mg} / \mathrm{kg}$ of sodium pentobarbitone. The experimental femora were excised from 6 rabbits of the OT group, the implants removed and the bone fixed in a solution of $10 \%$ neutral buffered formalin. Subsequently, they underwent $\mu-\mathrm{CT}$ analysis at the Institute of Health and Biomedical Innovation at the Queensland University of Technology. 1000 slices of the callus and fracture gap were taken over a $20 \mathrm{~mm}$ area using

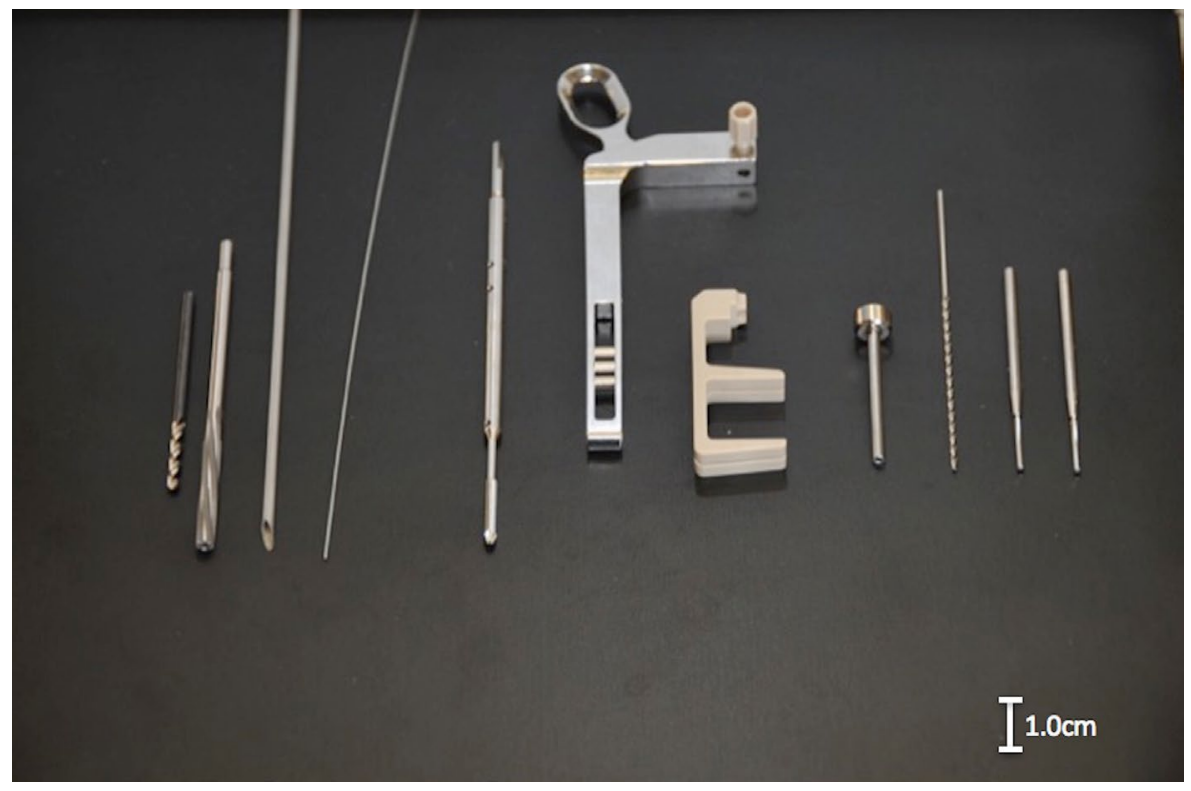

Fig. 4. RabbitNail and its surgical instruments. From left to right: small reaming drill bit $(1.2 \mathrm{~mm})$, large reaming drill bit $(2.8 \mathrm{~mm})$, guidewire, RabbitNail, external guide device (it attached to the RabbitNail as described in Fig. 1), osteotomy-guide device, locking screw sleeve, drill bit for locking screw $(1 \mathrm{~mm})$ and locking screws. 
Table 1: Radiographic scoring system (out of 10).

\begin{tabular}{|c|c|c|}
\hline Categories & Description & Score \\
\hline \multirow{4}{*}{$\begin{array}{c}\text { Periosteal reaction } \\
\text { (callus formation) }\end{array}$} & Full & 4 \\
\cline { 2 - 3 } & Moderate $(>1 / 2$ but $<3 / 4)$ & 3 \\
\cline { 2 - 3 } & Medium (extends over gap $>1 / 4)$ & 2 \\
\cline { 2 - 3 } & Minimal (localised to gap) & 1 \\
\hline \multirow{4}{*}{ Bone union } & None & 0 \\
\cline { 2 - 3 } & Union & 3 \\
\cline { 2 - 3 } & Moderate bridge $(>50 \%)$ & 2 \\
\cline { 2 - 3 } & Mild bridge $(<50 \%)$ & 1 \\
\hline \multirow{4}{*}{ Remodelling } & None & 3 \\
\cline { 2 - 3 } & Advanced remodelling & 1 \\
\cline { 2 - 3 } & Moderate remodelling & 0 \\
\cline { 2 - 3 } & Early remodelling & 2 \\
\hline
\end{tabular}

a $\mu$-CT 40 scanner (Scanco Medical, Bruttisellen, Switzerland) and Scanco Medical proprietary scanning/evaluation software. Two analyses were conducted: the first at a low threshold (468 mgHA/ $\mathrm{cm}^{3}$ ) to detect all mineralised tissue and the second at a high threshold $\left(1065 \mathrm{mg} \mathrm{HA} / \mathrm{cm}^{3}\right)$ to detect only old cortical bone. These analyses enabled a calculation of the callus volume and diameter.

\section{Lung and femur histology}

All 6 non-survival rabbits in the CF group were euthanised with intravenous injection of pentobarbitone, after the introduction of a high concentration of isoflurane. Immediately after death was confirmed, the lungs were harvested and placed in $10 \%$ neutral buffered formalin solution. Preferential sampling of the upper lobes of the lung was performed, to avoid the maximal areas of congestion occurring with death. A block was fixed in $10 \%$ formalin for $6-12 \mathrm{~h}$. Once the tissue was fixed, it was cut into $1 \times 1 \mathrm{~cm}$ sections. Next, the tissue was subjected to a series of increasing ethanol concentrations, from 70 to $100 \%$, for dehydration and xylene for clearing. Finally, the tissue was embedded in paraffin wax and cut using a Leica RM2255 (Leica, Berlin, Germany) microtome into $4 \mu \mathrm{m}$-thick sections. Deparaffinised sections were then stained with haematoxylin and eosin (H\&E). Oil red O staining was also performed on both fresh and fixed lung tissue to help determine the presence of fat embolisms.

After euthanasia at 8 weeks, femora of 6 rabbits of the OT group underwent a blind histological analysis by a pathologist at the Hunter Area Pathology Service based at John Hunter Hospital, Newcastle. Bones were decalcified by first fixing them in $10 \%$ formalin for $24 \mathrm{~h}$, then the specimens were kept for $48 \mathrm{~h}$ to $7 \mathrm{~d}$ in decalcification solution (5\% nitric oxide) to soften the tissue. The duration was determined by the pathologists and depended on the amount and nature of bone tissue present in the sample. Next, samples were embedded in paraffin wax and cut at $4 \mu \mathrm{m}$ thickness, before being stained with $\mathrm{H} \& \mathrm{E}$ and examined microscopically. Histological parameters used are summarised in Table 2 and were selected in consultation with an anatomical pathologist to provide the most thorough evaluation of fracture healing at 8 weeks. Furthermore, parameters were selected to align with the consensus view for histological evaluation of outcome in small animal fracture models (Histing et al., 2011). In detail, the parameter total bone volume was the percentage area of a histological slide of the callus, taken at the edge of the osteotomy site, that was occupied by any type of bone. The percentages of woven bone, cortical bone, trabecular bone, osteoid, vascular proliferation and fibrosis per $\mathrm{mm}^{2}$ indicated the average quantity of each one at the callus. Osteocyte necrosis, osteoblast number, osteoclast number and inflammatory cells were the number of each present per high-power field (hpf) at the osteotomy site (Olympus BX53 40× objective; Olympus, Sydney, Australia).

\section{Mechanical testing}

8 experimental femora from 4 rabbits of the OT group underwent biomechanical testing at the Australian School of Advanced Medicine, Macquarie University, NSW, Australia. The ends of each femur were potted in concentric plastic cylindrical tubes using an epoxy resin. The proximal end of the bone was fixed to the cross-head of an Instron mechanical testing machine (ElectroPuls E10000, Instron, Illinois) and the distal end placed on a sliding $X-Y$ table. A torsional moment was applied at a rate of $3 \% \mathrm{~s}$, until failure. The parameters tested were torsional stiffness, maximum torque, rotation at fracture, total energy and modulus of rigidity. The results for the fractured, healing femora were compared with those of the contralateral, unfractured femora. 
Table 2: Histological measures of bone healing.

\begin{tabular}{|c|c|}
\hline Non-cellular measures & Cellular measures \\
\hline Woven bone $(\%)$ & Osteocyte necrosis $(/ \mathrm{hpf})$ \\
\hline Cortical bone $(\%)$ & Osteoblast number $(/ \mathrm{hpf})$ \\
\hline Osteoid $(\%)$ & Osteoclast number $(/ \mathrm{hpf})$ \\
\hline Fibrosis $(\%)$ & Inflammatory cell $(/ \mathrm{hpf})$ \\
\hline & Vascular proliferation $(\%)$ \\
\hline
\end{tabular}

\section{Statistics}

Data were analysed using SPSS Statistics $17.00^{\circledR}$ (IBM, SPSS Statistics for Macintosh, Version 17.0). All variable and parameters were presented as mean \pm standard error. A Student's t-test was applied to paired data. Statistical significance was determined by a $p$ value of less than or equal to 0.05 . Adjustments for multiple comparisons was not required.

\section{Results}

A total of 6 non-survival rabbits and 17 rabbits in the chronic survival model ( $n=3$ in CF, $n=14$ in OT) were examined. The fracture patterns in CF were type A2, B1 and B2 of AO classification (Muller, 1980). Type A3 fracture was confirmed in all rabbits of the OT group. The rotational, torsional and axial stabilities were determined by manual examination at the conclusion of the procedure and the alignment and configuration were determined in post-operative X-rays (Fig. 5 and Fig. 6). There were no intra-operative complications related to the procedures. All rabbits were able to mobilise with partial/full weight bearing immediately

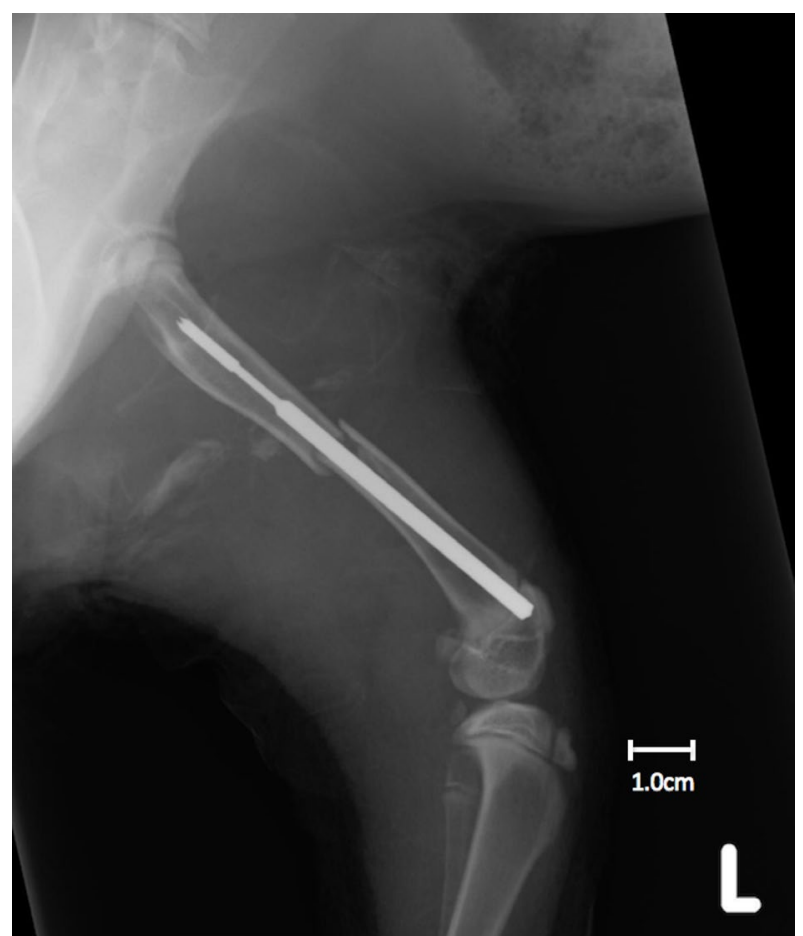

Fig. 5. X-ray image of RabbitNail in situ. The position of the nail was radiologically confirmed immediately after the fixation. after surgery. Full-weight bearing was achieved within $7 \mathrm{~d}$, with uneventful wound healing.

Significant periosteal reaction and callus formation was confirmed in all rabbits from 2 weeks postoperatively, with the radiological score of $1.0 \pm 0.1$ in the OT group and $3.6 \pm 0.1$ in the CF group. The healing had progressed toward the end of the experimental period in both groups. X-ray scores at 8 weeks were $9.6 \pm 0.2$ in the OT group $(p<0.05$ compared within the group) and $8.3 \pm 0.4$ in the CF group ( $p<0.05$ compared within the group). $\mu-\mathrm{CT}$ scans demonstrated extensive callus formation $\left(739.59 \pm 2.14 \mathrm{~mm}^{3}\right)$ and diminished mineral density at 8 weeks $\left(972.30 \pm 37.83 \mathrm{mgHa} / \mathrm{cm}^{3}\right.$ in callus and $1187.20 \pm 6.3183 \mathrm{mgHa} / \mathrm{cm}^{3}$ in control, $p<0.05$ ) (Fig. 7).

The histology of the lung samples demonstrated injury related to lung oedema in all rabbits. In addition, 2 (33\%) of rabbits demonstrated pulmonary emboli and thrombi (Fig. 8). Histology of 6 femora from the OT group were examined. As demonstrated radiologically, a mixture of woven bone $(20.0 \pm 10.3 \%)$, cortical bone $(17.5 \pm 3.1 \%)$ and trabecular bone $(11.6 \pm 2.1 \%)$ was mainly observed in the specimens, suggesting an early stage of remodelling (Fig. 9a,b). Inflammation was observed with associated vascular proliferation (5 $55 \%)$ and active healing process was confirmed by osteoblast and osteoclast count $(5.8 \pm 0.8 / \mathrm{hpf}$ and $1.1 \pm 0.8 / \mathrm{hpf}$, respectively).

At 8 weeks, biomechanical studies were performed on the osteotomised and contralateral femora of 4 animals, total 8 femora. The maximum torque and total energy required to break the femur demonstrated no difference between the osteotomised and contralateral control femora $(3.47 \pm 0.35 \mathrm{~N} \mathrm{~m}$ vs. $3.26 \pm 0.37 \mathrm{~N} \mathrm{~m}, 21.11 \pm 3.09 \mathrm{~N} \mathrm{~m} \times$ degree $v s$. $20.89 \pm 2.63 \mathrm{~N} \mathrm{~m} \times$ degree, respectively). Nevertheless, the modules of rigidity were significantly lower in the osteotomised femora compared with the contralateral control $(0.35 \pm 0.06 \mathrm{GPa}$ vs. $1.10 \pm 0.09 \mathrm{GPa}$, respectively, $p<0.05)$.

\section{Discussion}

Several animal models with femur fracture have been established to investigate its surgical treatment, immunological responses, impact of damage control surgery in polytrauma and general fracture healing. Small animal models such as rats, mice and rabbits, 


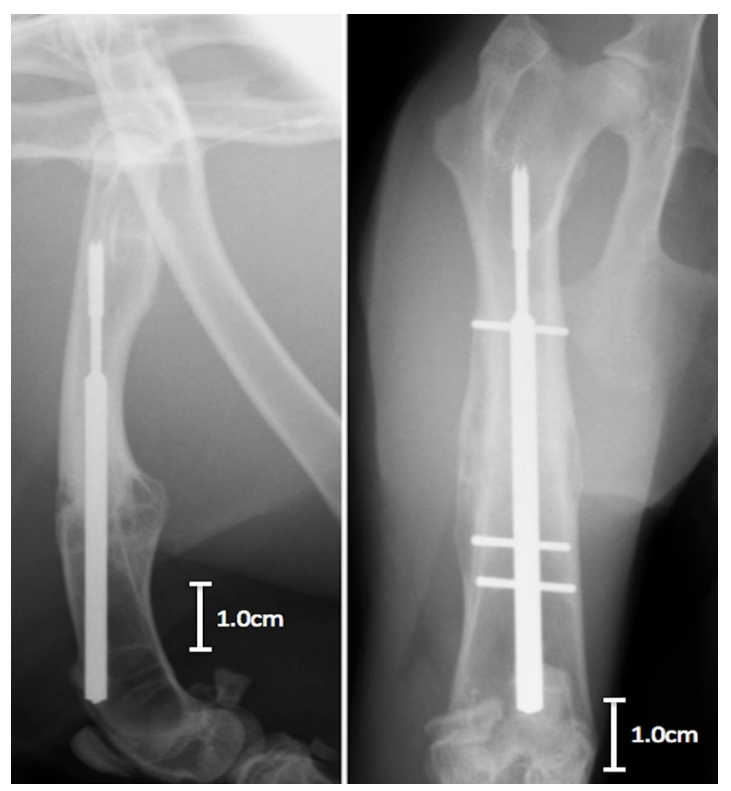

Fig. 6. (Left) 8 weeks postoperative X-ray, (right) 8 weeks postoperative $X$-ray image in an osteomised subject.

are less demanding financially and logistically and the recent/historical utilisation of animal models in bone studies is rat $(38 \%)$, rabbit $(19 \%)$ and mice (15\%) (O'Loughlin et al., 2008). Nevertheless, there are limitations of applicability to the human scenario, due to size, type of bone, different mechanisms of fracture healing, immunology, mechanical strength and brittleness of bone in the various models. The rabbit is a relatively small animal and it has been frequently used in physiology, cardiovascular and orthopaedic research (Arens et al., 2015; Chalmers et al., 1967; Keramaris et al., 2008; Kirlum et al., 2005; Terajima et al., 2006; Tiansheng et al., 2009; Wang et al., 2008). Its size enables researchers to conduct the preparation easier without large facilities and accommodation. The anatomy of the thigh, due to the abundant muscle coverage, may permit standardised soft tissue injury and optimal healing of the femur process (Wang et al., 2008). More importantly, the fracture-healing mechanism in a rabbit differs from mice and rats and might be more similar to that of the human, as it occurs with greater involvement of the medullary callus (Histing et al., 2011; O'Loughlin et al., 2008; Shirley et al., 2005). The femur fracture model was selected because of the clinical relevance of its systemic inflammatory and haemodynamic responses and because its stabilisation is essential for early mobilisation and recovery. This is a thoughtfully and purposefully selected model, which is relevant to major trauma-associated organ dysfunctions, including lung injury, as well as, impaired bone healing and post-injury stress response.

In this small animal model, it is satisfactorily demonstrated that the intramedullary nailing technique with RabbitNail can provide the necessary immediate stability and subsequent healing process among various types of femur fractures. The intramedullary nailing technique is a well-accepted standard technique for femur fracture, which allows adequate skeletal reconstruction with early mobilisation and adequate healing with low non-union rate, although it might cause other complications.

Acute complications were observed in this model, including bone marrow embolus, as well as, inflammation and oedema in the lung. A standardised internal fixation method for femur fracture, reamed and locked intramedullary nailing technique, has been rarely applied to small animal models due to the lack of availability of suitable devices and difficulties related to animal size. In previously published studies, investigator-modified Kirschner wires or plate fixation techniques were often employed (Heim et al., 1995; Krishnan et al., 2015; Tiansheng et al., 2009) and the physiological impact and consequences of the interventions were often similar to those observed in the clinical scenario from femur fracture and its internal fixation (Reuter et al., 2002; Wang et al., 2008). As the femur is a weight-bearing bone, the axial, rotational and torsional instability could alter the fracture healing process. Internal fixation with RabbitNail resembles the current technique used in human practice with more clinically relevant outcomes and complications. Given the limitation of the intra-operative applicability of fluoroscopy in small animal laboratory setting, the guide-wire technique was applied and the external aiming device permitted us to insert, with accuracy, the locking screws percutaneously. This technique with osteotomy is readily applied for non-union animal model since the proximal and distal insertions of the guided locking screws can precisely determine the size of gap between fractured bones.

The intramedullary nail was durable enough to create immediate axial and torsional stability to allow functional recovery and timely fracture healing with abundant callus formation (Fig. 6). The healing with RabbitNail was consistently commenced within 2 weeks, regardless of the types of fractures. Subsequent healing was also demonstrated radiologically and histologically with the suggestion of early remodelling at 8 weeks postoperatively. The modulus of rigidity indicated that the callus at 8 weeks was significantly more elastic than the contralateral control femur. Nevertheless, the overall mechanical strength observed at 8 weeks was similar to that of the control. This was more expeditious than those of other previously applied techniques (Carrillo et al., 2005; Kettunen et al., 1999).

Reproducibility of the fracture patterns in rabbits of CF group has not been successfully demonstrated. Nevertheless, RabbitNail produced a reproducible clinical outcome: full mobilisation within $2 \mathrm{~d}$. Durability and strength of the nail were well demonstrated in all rabbits. The RabbitNail helped the mobilisation of the rabbits and led to subsequent healing in both CF and OF groups, which were all consistent and applicable to the clinical scenario of femur fractures. 


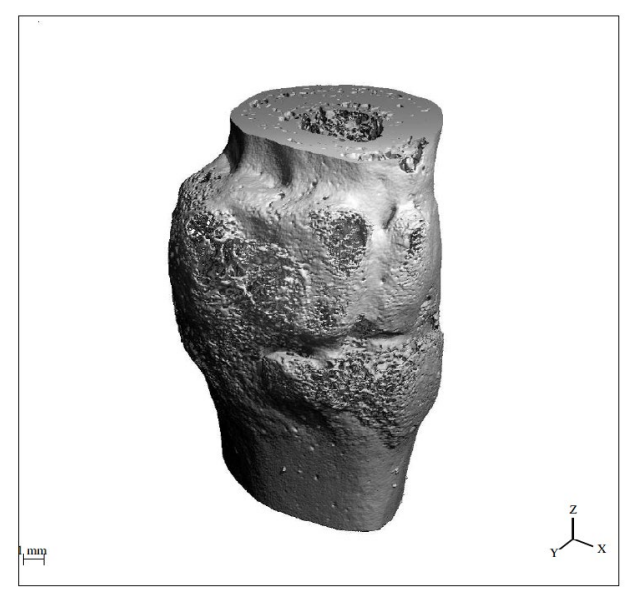

Fig. 7. Three-dimensional reconstruction of the 8 weeks postoperative $\mu$-CT scan of the fracture callus.

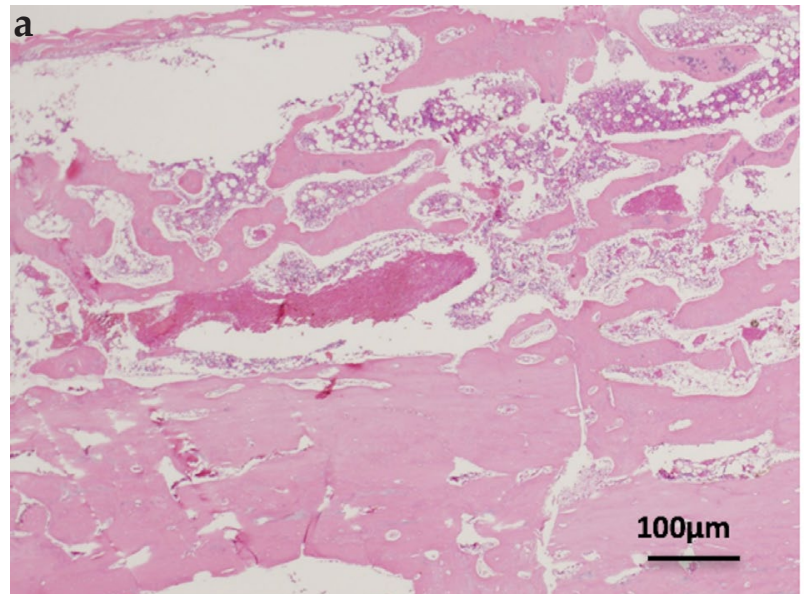

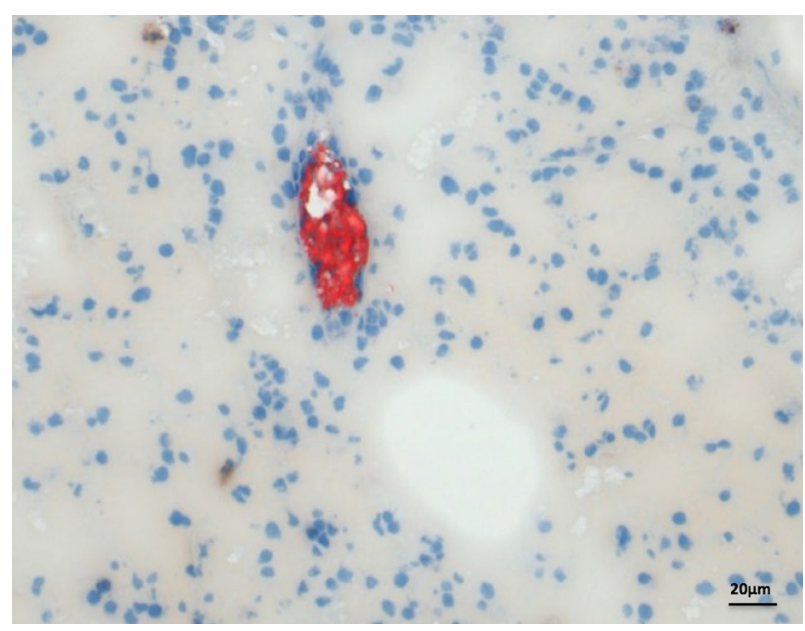

Fig. 8. Fat embolism observed in lung.

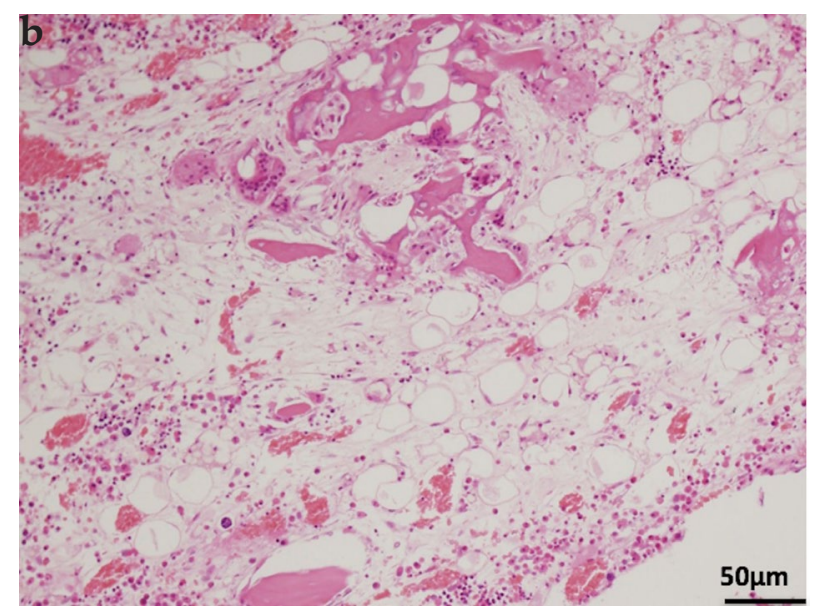

Fig. 9. Histology consistent with early stage of remodelling including fibrosis and neovascularisation at the fracture site (a) and osteoid formation at the fracture site $(\mathbf{b})$.

\section{Conclusion}

Standardised internal fixation technique with reamed and locked intramedullary nail (RabbitNail) for a closed fracture and a femur osteotomy was developed. This is a clinically relevant technique, which provides uneventful healing. The model demonstrated histologically, biomechanically and radiologically bone healing with clinically-relevant complications, including pulmonary embolus. Potentially, studies related to fracture healing, intramedullary nail technique and its effects on physiology can be conducted with RabbitNail.

\section{References}

Al-Arabi YB, Nader M, Hamidian-Jahromi AR, Woods DA (2007) The effect of the timing of antibiotics and surgical treatment on infection rates in open long-bone fractures: a 9-year prospective study from a district general hospital. Injury 38: 900-905.

Arens D, Wilke M, Calabro L, Hackl S, Zeiter S, Zderic I, Richards RG, Moriarty TF (2015) A rabbit humerus model of plating and nailing osteosynthesis with and without Staphylococcus aureus osteomyelitis. Eur Cell Mater 30: 148-162.

Balogh ZJ, Reumann MK, Gruen RL, MayerKuckuk P, Schuetz MA, Harris IA, Gabbe BJ, Bhandari M (2012) Advances and future directions for management of trauma patients with musculoskeletal injuries. Lancet 380: 1109-1119.

Bonnarens F, Einhorn TA (1984) Production of a standard closed fracture in laboratory animal bone. J Orthop Res 2: 97-101.

Carrillo JM, Sopena JJ, Rubio M, Redondo JI, Serra I, Soler C (2005) Experimental use of polyamide bands in combination with intramedullary pinning for repair of oblique femoral fractures in rabbits. Vet Surg 34: 387-392.

Chalmers JP, Korner PI, White SW (1967) The effects of haemorrhage in the unanaesthetized rabbit. J Physiol 189: 367-391.

Court-Brown CM, Rimmer S, Prakash U, McQueen MM (1998) The epidemiology of open long bone fractures. Injury 29: 529-534.

Forster MC, Aster AS, Ahmed S (2005) Reaming during anterograde femoral nailing: is it worth it? Injury 36: 445-449. 
An YH, Friedman RJ, Draugh RA (1999). Animal models of bone fracture or osteotomy. In: An YH and Friedman RJ, Ed. Animal models in orthopedic research. CRC Press, Boca Raton, Florida, USA, Part III 11: 197-218.

Hardy BM, Yoshino O, Quail AW, Balogh ZJ (2015) Influence of the timing of internal fixation of femur fractures during shock resuscitation on remote organ damage. ANZ J Surg 85: 966-971.

Heim D, Regazzoni P, Tsakiris DA, Aebi T, Schlegel U, Marbet GA, Perren SM (1995) Intramedullary nailing and pulmonary embolism: does unreamed nailing prevent embolization? An in vivo study in rabbits. J Trauma 38: 899-906.

Histing T, Garcia P, Holstein JH, Klein M, Matthys R, Nuetzi R, Steck R, Laschke MW, Wehner T, Bindl R, Recknagel S, Stuermer EK, Vollmar B, Wildemann B, Lienau J, Willie B, Peters A, Ignatius A, Pohlemann T, Claes L, Menger MD (2011) Small animal bone healing models: standards, tips, and pitfalls results of a consensus meeting. Bone 49: 591-599.

Johnson KD, Frierson KE, Keller TS, Cook C, Scheinberg R, Zerwekh J, Meyers L, Sciadini MF (1996) Porous ceramics as bone graft substitutes in long bone defects: a biomechanical, histological, and radiographic analysis. J Orthop Res 14: 351-369.

Keramaris NC, Calori GM, Nikolaou VS, Schemitsch EH, Giannoudis PV (2008) Fracture vascularity and bone healing: a systematic review of the role of VEGF. Injury 39 Suppl 2: S45-57.

Kettunen J, Makela A, Miettinen H, Nevalainen T, Heikkila M, Tormala P, Rokkanen P (1999) Fixation of distal femoral osteotomy with an intramedullary rod: early failure of carbon fibre composite implant in rabbits. J Biomater Sci Polym Ed 10: 715-728.

Kirlum HJ, Heinrich M, Till H (2005) The rabbit model serves as a valuable operative experience and helps to establish new techniques for abdominal and thoracic endosurgery. Pediatr Surg Int 21: 91-93.

Krishnan L, Priddy LB, Esancy C, Li MT, Stevens HY, Jiang X, Tran L, Rowe DW, Guldberg RE (2015) Hydrogel-based delivery of rhBMP-2 improves healing of large bone defects compared with autograft. Clin Orthop Relat Res 473: 2885-2897.

Ktistakis I, Giannoudi M, Giannoudis PV (2014) Infection rates after open tibial fractures: are they decreasing?. Injury 45: 1025-1027.

Lasanianos NG, Kanakaris NK, Dimitriou R, Pape HC, Giannoudis PV (2011) Second hit phenomenon: existing evidence of clinical implications. Injury 42: 617-629.

Muller ME (1980) [Classification and international AO-documentation of femur fractures]. Unfallheilkunde 83: 251-259.

O'Loughlin PF, Morr S, Bogunovic L, Kim AD, Park B, Lane JM (2008) Selection and development of preclinical models in fracture-healing research. J Bone Joint Surg Am 90 Suppl 1: 79-84.

Reuter JD, Ovadia S, Howell P, Jaskwich DH (2002) Femoral fracture repair and postoperative management in New Zealand white rabbits. Contemp Top Lab Anim Sci 41: 49-52.

Shirley D, Marsh D, Jordan G, McQuaid S, Li G (2005) Systemic recruitment of osteoblastic cells in fracture healing. J Orthop Res 23: 1013-1021.

Tay WH, de Steiger R, Richardson M, Gruen R, Balogh ZJ (2014) Health outcomes of delayed union and nonunion of femoral and tibial shaft fractures. Injury 45: 1653-1658.

Terajima K, Tsueshita T, Sakamoto A, Ogawa R (2006) Fluid resuscitation with hemoglobin vesicles in a rabbit model of acute hemorrhagic shock. Shock 25: 184-189.

Tiansheng S, Xiaobin C, Zhi L, Xiaowei W, Guang L, Liren Z (2009) Is damage control orthopedics essential for the management of bilateral femoral fractures associated or complicated with shock? An animal study. J. Trauma 67: 1402-1411.

Wang CJ, Wang FS, Yang KD (2008) Biological effects of extracorporeal shockwave in bone healing: a study in rabbits. Arch Orthop Trauma Surg 128: 879-884.

White TO, Clutton RE, Salter D, Swann D, Christie J, Robinson CM (2006) The early response to major trauma and intramedullary nailing. J Bone Joint Surg Br 88: 823-827.

Yang CY, Simmons DJ, Lozano R (1994) The healing of grafts combining freeze-dried and demineralized allogeneic bone in rabbits. Clin Orthop Relat Res 298: 286-295.

Editor note: All of the matters raised by the reviewers have been incorporated in the main text, so there is no Discussion with reviewers section for this paper.

The scientific editor for this paper was Chris Evans. 